\title{
Plasmodium falciparum gametocyte carriage, sex ratios and asexual parasite rates in Nigerian children before and after a treatment protocol policy change instituting the use of artemisinin-based combination therapies
}

\author{
Grace Olusola Gbotosho, Akintunde Sowunmi ${ }^{+}$, \\ Christian Tientcha Happi, Titilope Modupe Okuboyejo
}

\author{
Department of Pharmacology and Therapeutics and Malaria Research Laboratories, \\ Institute for Medical Research and Training, University of Ibadan, Ibadan, Nigeria
}

The effects of artemisinin-based combination therapies (ACTS) on transmission of Plasmodium falciparum were evaluated after a policy change instituting the use of ACTs in an endemic area. P. falciparum gametocyte carriage, sex ratios and inbreeding rates were examined in 2,585 children at presentation with acute falciparum malaria during a 10-year period from 2001-2010. Asexual parasite rates were also evaluated from 2003-2010 in 10,615 children before and after the policy change. Gametocyte carriage declined significantly from $12.4 \%$ in 2001 to $3.6 \%$ in 2010 $(\chi$ for trend $=44.3, p<0.0001$ ), but sex ratios and inbreeding rates remained unchanged. Additionally, overall parasite rates remained unchanged before and after the policy change (47.2\% vs. $45.4 \%)$, but these rates declined significantly from 2003-2010 ( $\chi$ for trend 35.4, $p<0.0001$ ). Chloroquine $(C Q)$ and artemether-lumefantrine $(A L)$ were used as prototype drugs before and after the policy change, respectively. AL significantly shortened the duration of male gametocyte carriage in individual patients after treatment began compared with CQ (log rank statistic $=7.92, p=0.005)$. ACTs reduced the rate of gametocyte carriage in children with acute falciparum infections at presentation and shortened the duration of male gametocyte carriage after treatment. However, parasite population sex ratios, inbreeding rates and overall parasite rate were unaffected.

Key words: P. falciparum - gametocyte carriage - sex ratio - parasite rate - artemisinin drugs - children

Artemisinin-based combination therapies (ACTs) are recommended as first-line treatments for Plasmodium falciparum malaria globally because they rapidly reduce the asexual parasite biomass and gametocyte carriage. Subsequently, these treatments reduce the chances that P. falciparum will develop drug resistance (WHO 2001). In areas of low transmission, especially on the western border of Thailand, ACTs have consistently reduced transmission and are thought to have broken the chains of transmission (Price et al. 1996). Over 50 African countries where transmission is endemic have adopted ACTs as the first-line treatment protocol (WHO 2010). After adopting the treatment protocol policy change to ACTs, it is unclear whether the use of ACTs has objec-

Financial support: Swiss Pharma Nigeria (PLC to AS), FIRCA (NIH RO3TW007757-02) (to CTH), IAEA (project RAF/0625), UNI$\mathrm{CEF} / \mathrm{UNDP} /$ WorldBank/WHO/TDR/PAG/South-South Initiative (ID A50337), Harvard Malaria Initiative, EDCTP (TA2007/40200016) for Senior Research Fellowship, MIM/TDR (ID A20239) (to GOG)

GOG and AS led the design, conduct, data analysis and manuscript preparation. TMO and $\mathrm{CTH}$ were involved in design, conduct and data analysis. +Corresponding author: akinsowunmi@hotmail.com

Received 7 February 2011

Accepted 19 July 2011 tively influenced transmission in these countries in any meaningful way; although when compared to the use of non-ACTs, they have reduced the transmission of $P$. falciparum from individual patients with uncomplicated infections to mosquitoes (Okell et al. 2008).

The uptake from human blood of viable male and female $P$. falciparum gametocytes and their subsequent development into gametes in the mosquito vector are crucial to malaria transmission (Sinden 1983, Carter \& Graves 1988). Mosquito infectivity after a human blood meal and the subsequent transmission is thought to be dependent on gametocyte density (Tchuinkam et al. 1993, Robert et al. 1996, Ouédraogo et al. 2009) and may be influenced by the gametocyte sex ratios (GSR) in the blood meal (Robert et al. 1996, Mitri et al. 2009). It has been shown that the proportion of infected mosquitoes and the mean oocyst load increased as sex ratio in the infected human blood meals increased towards 0.5 (Robert et al. 1996). In cultured gametocytes, increasing the male sex ratio increased the mosquito infectivity rate at low levels of gametocyte density, whereas there was a reduced infectivity rate at higher levels of gametocyte density (Mitri et al. 2009). ACTs reduce gametocyte carriage and transmission of $P$. falciparum to mosquitoes (Okell et al. 2008). However, it is unclear whether ACTs affect the gametocyte population sex ratios or the inbreeding rates enough to influence transmission when introduced as first-line treatments in endemic settings. 
In addition, the effects of ACTs on the duration of male gametocyte carriage after treatment are unknown. Such information would be of great value in improving community control of malaria in endemic settings.

To address the above questions, gametocyte carriage, sex ratios and inbreeding rates were evaluated at presentation in children with acute, symptomatic and apparently uncomplicated falciparum malaria. These evaluations were performed in the four and a half years before and the five and a half years after the May 2005 treatment protocol policy change instituting the use of ACTs [artemetherlumefantrine (AL) and artesunate-amodiaquine] in an endemic area of intense transmission (FMH 2005). In addition, asexual parasite rates were evaluated for five years in 10,615 children before and after the policy change. The effects of two prototype drugs, chloroquine (CQ), used before the policy change, and AL, used after the change, on the duration of male gametocyte sex ratio in individual patients after treatment began were also evaluated.

\section{PATIENTS, MATERIALS AND METHODS}

The study consisted of two parts: (i) the evaluation of gametocyte carriage, sex ratios and inbreeding rates and the effects of the prototype antimalarial drugs on the duration of male gametocytes following treatment, both before and after the policy change instituting the use of ACTs, and (ii) the assessment of asexual parasite rates in children with febrile illness before and after the policy change instituting the use of ACTs (Fig. 1).

Patients enrolled in drug studies - Patients were recruited into antimalarial efficacy studies as described previously (Sowunmi et al. 2009a), from January 2001November 2010, at The Malaria Clinic of the University College Hospital in Ibadan, a malaria-endemic area in southwest Nigeria (Salako et al. 1990). All patients with a presumptive diagnosis of malaria were evaluated for the presence of $P$. falciparum parasitaemia. Patients were enrolled if they met the following conditions: their attending relatives gave informed consent, they were between 0.5-15 years old, they had symptoms compatible with malaria, they had single species asexual P. falciparum parasitaemia $>2,000 / \mu \mathrm{L}$ of blood, they did not have a history of significant antimalarial drug intake in the two weeks preceding presentation and they had a strong likelihood of being able to complete the four-six weeks of follow-up. Patients with severe malaria (WHO 2000), serious underlying diseases (renal, cardiac or hepatic) or severe malnutrition were excluded from the study. Patients were randomised to receive CQ (pre-policy change, i.e., up to 2004) or AL (at policy and post policy change, i.e., 2005 and after). The studies received approval from the local ethics committee.

The attending physician recorded the disease history by asking patients or their parents or guardian when the present symptomatic period began. The history was followed by a full physical examination by the same physician.

Clinical and parasitological follow-up evaluations were carried out daily on days 1-7 and then on days $14,21,28,35$ and 42 . The follow-up inquired about the patient's well-being, the presence or absence of initial presenting symptoms, the presence of additional symptoms, measurement of body temperature, heart and respiratory rates and a blood smear for the quantification of peripheral parasitaemia.

Laboratory investigations - Assuming a leukocyte count of $6,000 / \mu \mathrm{L}$ of blood, quantification in Giemsastained thick and thin blood films was done against 500 leukocytes in the case of asexual parasites and against 1,000 leukocytes in the case of sexual parasites. Gametocytes were sexed if gametocytaemia was $>10 / \mu \mathrm{L}$ blood. Gametocyte sex determination was based on the following criteria (Carter \& Graves 1988): males (microgametocytes) are smaller than females (macrogametocytes), the nucleus is larger in males than females, the ends of the cells are rounder in males and angular in females, with Giemsa, the cytoplasm stains purple in males and deep blue in females and the granules of malaria pigment are centrally located in females and more widely scattered in males. Gametocytes were classified morphologically as male or female if at least three of the five above criteria were present. The sex ratio was defined as the proportion of gametocytes in peripheral blood that were male (Pickering et al. 2000, West et al. 2001, 2002). The inbreeding rate was calculated using the equation, $f=1-2 z^{*}$ (Nee et al. 2002), where $\mathrm{z}^{*}$ is the proportion of male to female gametocytes.

Capillary blood drawn before and during follow-up was used to measure packed cell volume or the haematocrit. The haematocrits were measured using a microhaematocrit tube and microcentrifuge (Hawksley, Lancing, UK). Routine haematocrit was measured on days

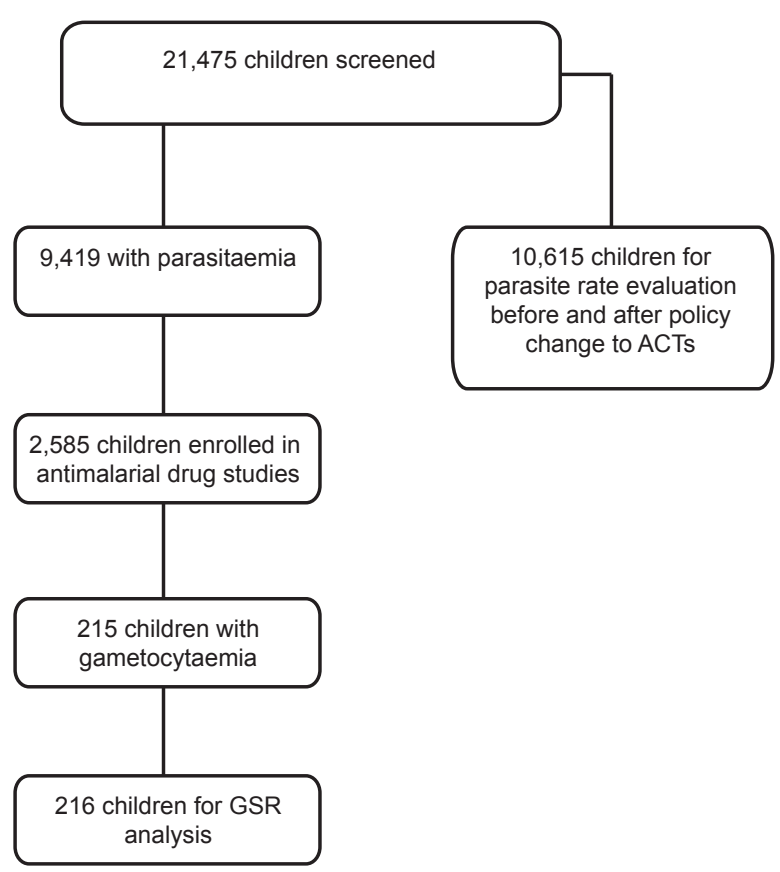

Fig. 1: study profile. ACTs: artemisinin-based combination therapies; GSR: gametocyte sex ratios. 
$0-7,14,21,28$ and, when possible, on days 35 and 42 . The effects of the policy change to ACT use on malariaassociated anaemia will be reported in future research.

Asexual parasite rate - All 15 year-old or younger children with symptoms suggestive of malaria were initially enrolled in the study. Blood was obtained for parasite screening and species identification in all children using the methods described above. However, analysis of $P$. falciparum parasite positivity rate was confined to children enrolled in the study in 2003, 2004, 2005, 2009 and 2010.

Data analysis - Data were analysed using version 6 of the Epi-Info software (CDC 1994) and the statistical programme SPSS for Windows version 10.01 (SPSS 1999). The analysis considered variables related to the densities of $P$. falciparum gametocytes and trophozoites. Proportions were compared by calculating $\chi^{2}$ with Yates' correction or by the Fisher exact test. Normally distributed, continuous data were compared by the Student's $t$ test and analysis of variance. Data not conforming to a normal distribution were compared by the Mann-Whitney $U$-tests, the Kruskal-Wallis tests, or the Wilcoxon ranked sum test. Kaplan-Meier plots are also presented to compare male gametocyte carriage rates after treatment in patients who were gametocytaemic at presentation. All tests of significance were two-tailed. $p$ values of $<0.05$ were taken to indicate significant differences. Data were (double)-entered serially using the patients' codes and were only analysed at the end of the study.

\section{RESULTS}

Patients' characteristics at enrolment - Over the course of the study period, 2,585 children (1,497 before the policy change instituting the use of ACTs and 1,088 after) were evaluated at presentation with acute falciparum malaria. The characteristics of these children are summarised in Table. Children enrolled before the policy change were significantly younger than children enrolled after the policy change instituting the use of ACTs primarily because change antimalarial efficacy studies prior to the policy change were conducted mostly with children under five years old. Additionally, these younger children had significantly lower parasitaemia and gametocytaemia.

Gametocyte carriage at presentation with acute falciparum infections - Overall gametocyte carriage in children at presentation for acute malaria was $9.4 \%(148 / 1,497)$ before the policy change and 6.2\% $(67 / 1,088)$ after $(p=$ $0.0009)$. Gametocyte carriage declined significantly from $12.4 \%$ in 2001 to $3.6 \%$ in 2010 ( $\chi^{2}$ for trend $=44.3, \mathrm{p}<$ 0.0001 ) (Fig. 2). Significant decreases were obvious from 2009, four years after policy change to ACTs.

Asexual parasite rates - The overall parasite rate during the 10 -year period was $43.8 \%(9,419 / 21,475)$ (Fig. 1). Of 9,419 children with positive smear slides, 9,325 children had mono infections with P. falciparum, 56 children had mixed infections with $P$. falciparum and

TABLE

Demographic and clinical characteristics of children enrolled four years before and six years after policy change to artemisinin-based combination therapies (ACTs) in an endemic area

\begin{tabular}{|c|c|c|c|}
\hline Variable & Before policy change to ACTs & After policy change to ACTs & $\mathrm{p}$ value \\
\hline Number of patients & 1,497 & 1,088 & - \\
\hline Male $[\mathrm{n}(\%)]$ & $713(47.6)$ & $571(52.4)$ & - \\
\hline Age (year) [mean (SD)] & $5.8(3.1)$ & $6.4(3.0)$ & 0.0001 \\
\hline Range & $0.3-15$ & $0.4-15$ & - \\
\hline$<5$ years $[\mathrm{n}(\%)]$ & $656(43.8)$ & $383(35.2)$ & 0.00001 \\
\hline Weight (kg) [mean (SD)] & $17.5(6.5)$ & $18.1(6.5)$ & 0.016 \\
\hline Range & $4-44$ & $6-59$ & - \\
\hline Hematocrit (\%) [mean (SD)] & $30.8(4.8)$ & $31.2(4.6)$ & 0.06 \\
\hline Range & $17-49$ & $13-48$ & - \\
\hline$<30 \%$ & $541(\mathrm{n}=1,449)$ & $296(n=926)$ & 0.008 \\
\hline$<25 \%[\mathrm{n}(\%)]$ & $130(8.9)$ & $74(7.9)$ & 0.45 \\
\hline Temperature $\left({ }^{\circ} \mathrm{C}\right)[$ mean $(\mathrm{SD})]$ & $38.2(1.10)$ & $38.4(1.1)$ & 0.001 \\
\hline Range & $35-41.1$ & $35.8-41.1$ & - \\
\hline$>40^{\circ} \mathrm{C}[\mathrm{n}(\%)]$ & $65(4.3)$ & $57(5.2)$ & 0.31 \\
\hline Duration of illness [mean (SD)] & $3.0(1.3)$ & $2.8(1.2)$ & 0.001 \\
\hline Range & $1-10$ & $1-10$ & - \\
\hline GMPD (/ $\mu \mathrm{L}$ blood $)$ & 32,708 & 52,012 & 0.0001 \\
\hline Range & $708-6,194,285$ & $837-2,124,000$ & - \\
\hline$>250,000[\mathrm{n}(\%)]$ & $107(7.1)$ & $86(7.9)$ & 0.79 \\
\hline GMGD (/ $\mu \mathrm{L}$ blood $)$ & $18(\mathrm{n}=148)$ & $34(\mathrm{n}=67)$ & 0.03 \\
\hline Range & 6-324 & 6-468 & - \\
\hline
\end{tabular}

GMGD: geometric mean gametocyte density; GMPD: geometric mean parasite density; SD: standard deviation. 
Plasmodium malariae, and 38 had mono infections with P. malariae. As many as 10,615 children were evaluated for parasite rate studies before $(\mathrm{n}=2,032)$ and after (n $=8,583$ ) the policy change. Parasitaemia was present in 960 children before and 3,900 children after the policy change instituting the use of ATCs. There was no significant difference in overall parasite rate before and after the policy change $\left(\chi^{2}=2.09, p=0.148\right)$. However, there was a significant decline in parasite rate between 20032010. Parasite rates were $577 / 1,087$ in $2003,383 / 945$ in $2004,1,399 / 2,864$ in $2005,1,629 / 3,504$ in 2009 and $872 / 2,215$ in 2010 ( $\chi^{2}$ for trend $\left.=35.4, \mathrm{p}<0.0001\right)$.

GSR and inbreeding rates at enrolment - Data were available for gametocyte sex ratio evaluation at presentation in 148 children before and in 67 children after policy change instituting the use of ACT. Upon enrolment, 4,896 gametocytes were sexed before and 3,099 gametocytes were sexed after the policy change. The weighted mean sex ratios were 0.21 [95\% confidence interval (CI) 0.16 $0.24]$ and 0.20 (95\% CI $0.15-0.25)$, respectively. The corresponding inbreeding rates were 0.58 (95\% CI 0.52-0.68) and $0.60(95 \% \mathrm{CI} 0.50-0.70)$, respectively. There were no significant differences in all the parameters before and after the policy change to instituting the use of ACTs.

Duration of male gametocyte carriage following treatment with $C Q$ and $A L$ - Data were available for analysis of the duration of male gametocyte carriage following treatment with the prototype drugs, CQ and AL in 28 patients. One of 14 children treated with AL and eight of 14 children treated with CQ had male gametocytaemia three days after commencement of treatment $(p=0.012)$. Kaplan-Meier plots of the probability of male gametocyte carriage demonstrated that the treatment with AL significantly shortens the duration of male carriage when compared with CQ $(\log$ rank statistic $=7.92, p=0.005)($ Fig. 3).

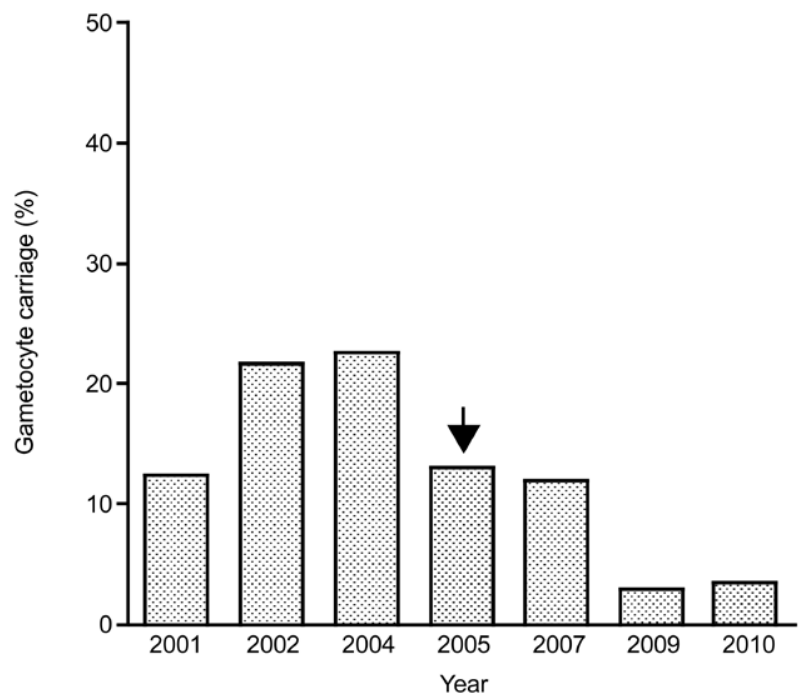

Fig. 2: gametocyte carriage at enrolment in children with acute falciparum malaria four years before and six years after policy change to artemisinin-based combination therapies in an endemic area. The arrowhead indicates the year of the policy change.

\section{DISCUSSION}

In the present study, gametocyte carriage at presentation in children with acute falciparum malaria declined significantly after a treatment protocol policy change instituting the use of ACTs in an endemic area of southwest Nigeria. Appreciable decline in carriage was obvious four years after the change in policy. The relative delay in the decline in carriage was partly due to the fact that widespread use of ACTs and the decline in use of CQ monotherapy occurred only recently (Mokuolu et al. 2007). The latter is supported by a finding that in 2006, $20 \%$ of children with acute falciparum malaria had detectable CQ concentrations in blood samples obtained at presentation (personal observation). In addition, despite the policy change, CQ was and is still readily available over the counter. Although gametocyte carriage rates are now lower after consistent and widespread use of ACTs in patients with acute malaria, these relatively low rates are likely to be underestimated due to significant submicroscopical gametocytaemia. The submicroscopical gametocytaemia in children from this and other endemic areas (Shekalaghe et al. 2007, Happi et al. 2009, Ouédraogo et al. 2009) is detectable by polymerase chain reaction (PCR) and may substantially contribute to the gametocyte reservoir.

Despite significant decreases in gametocyte carriage in children with acute malaria and a significant decline in asexual parasite rates, the overall post-policy change asexual parasite rate, a measure of transmission intensity, remains high at $45 \%$. The following are possible reasons for this high rate: (i) the presence in the community of a large reservoir of infection, particularly in the older children and adults, although there are no recent data on the reservoir of infection in adults in this community, (ii) submicroscopical gametocytaemia may be common in the community even after treatment with ACTs (Happi et al. 2009) and may be a source of mosquito infectivity [this is supported by a recent study showing that even in areas of low transmission, submicroscopical gametocytaemia may contribute substantially to the infectious

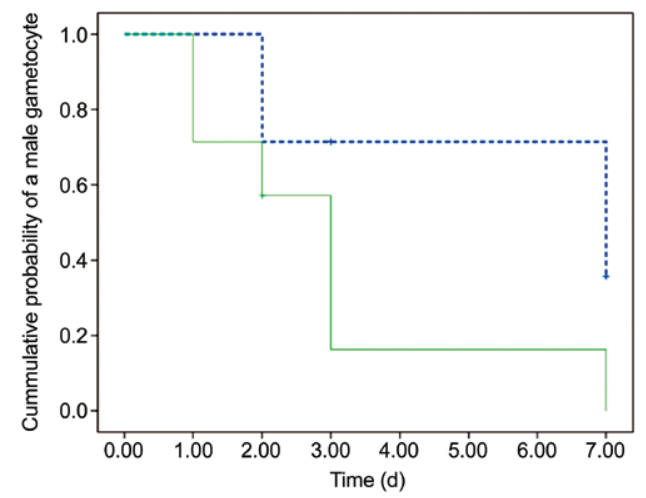

Fig. 3: Kaplan-Meier plot (survival curve) of the cumulative probability of carrying a male gametocyte after treatment with chloroquine (broken line) or artemether-lumefantrine (solid line) in children with Plasmodium falciparum infections $(\log$ rank statistic $=7.92, \mathrm{p}=0.005)$. 
reservoir (Ouédraogo et al. 2009)], (iii) frequent use of gametocytaemia enhancing antimalarial drugs (e.g., sulfadoxine-pyrimethamine) by asymptomatic carriers of infections for the treatment of illnesses presumed to be malaria and for intermittent preventive therapy of malaria during pregnancy and (iv) an efficient vector, Anopheles gambiae, and favourable conditions for mosquito breeding and infectivity.

In keeping with the fact that Plasmodium species sex ratios are often female biased (Robert et al. 1996, Pickering et al. 2000, West et al. 2000, 2001, 2002, Reece et al. 2008), the gametocyte sex ratio in our cohort of children was also female biased and differed little over a 10 -year period. The gametocyte sex ratio of $0.20-0.21$ suggests, theoretically, that this is the maximum degree of female bias expected if the average number of viable gametes released after exflagellation by one male gametocyte was four (Read et al. 1992, West et al. 2002). The population sex ratio in this endemic area is similar to that reported in neighbouring Cameroon [0.217 (Robert et al. 1996)], but it is lower than the 0.346 reported in Senegal (Robert et al. 2003). In this endemic area clone multiplicity in $P$. falciparum infections is approximately three-four (Happi et al. 2003, 2004, 2006) and this appears to have changed little over the years (Happi et al. 2003, 2004, 2006).

The inbreeding rates in the present study are similar to those found in another recent study (Sowunmi et al. 2009a, b). Considering that our time periods overlapped, this similarity was expected. The similar population sex ratios and breeding rates before and after the policy change suggest that ACTs had little or no effect on the gametocyte sex ratio in this endemic setting although ACTs tend to produce a female-biased sex ratio in the individual patients after treatment (Sowunmi et al. 2007, 2009a). The lack of the effect of ACTs on sex ratios and inbreeding rates would be expected since the individual patients receiving ACTs constitute a negligible portion of the parasite reservoir.

To assess the influence of the prototype drugs used before and after the policy change instituting the use of ACTs on sex ratios after treatment of individual patients, we estimated the duration of male gametocyte carriage using a Kaplan-Meier survival analysis. This approach has definite advantages in an endemic area where parasite population sex ratio is female biased (Sowunmi et al. 2009a, b). Mosquito infectivity and therefore transmission can only occur if male gametocytes are present in the blood meal obtained by the mosquito. The duration of mosquito infectivity, in the presence of female gametocytes is, theoretically, directly related to the duration of the concomitant male gametocyte and density. Using this approach, AL effectively shortened the duration of male gametocyte carriage in individual patients following treatment. If at low gametocytaemia, a male-biased sex ratio is crucial to malaria transmission (Robert et al. 1996, Mitri et al. 2009, Ouédraogo et al. 2009), then $\mathrm{AL}$ can be expected to reduce the duration of exposure to potentially successful mosquito infectivity following a blood meal by shortening the duration of male gametocyte carriage compared to CQ. Could this effect produced by AL or other ACTs significantly influence malaria transmission in this endemic area? It seems unlikely when observing the many factors contributing to high transmission in the area (see above).

The main limitations of the present study are as follows: symptomatic individuals were employed in the evaluation of the various gametocyte carriage rates, the various gametocyte carriage rates are likely to be underestimated since submicroscopic gametocytaemia detectable by PCR is not uncommon in children from endemic areas (Shekalaghe et al. 2007, Happi et al. 2009) and may contribute substantially to transmission, and gametocyte sex was determined morphologically, instead of using PCR, a more accurate method of gametocyte sex determination (Drew \& Reece 2007). In addition, gametocyte viability and infectivity to mosquito before and after policy change was not evaluated. However, an important public health implication of the findings is that prompt diagnosis and treatment with ACTs and the combination of ACTs use with other control measures is likely to further reduce gametocyte carriage and subsequent transmission in endemic areas.

In conclusion, ACTs reduce gametocyte carriage in children with acute falciparum infections at presentation and shorten the duration of male gametocyte carriage after treatment of individual patients, but parasite population sex ratios and inbreeding rates are unaffected. The asexual parasite rate, as a measure of transmission intensity, also declined significantly but remained high at $45 \%$ in this endemic area.

\section{ACKNOWLEDGEMENTS}

To our clinic staff, most especially Dr Stanley Michael, Omowunmi Lawal, Ebunsola Oketade, Abayomi Sijuade, Gbenga Akinola and Matthew Olatunde, for assistance with running the studies.

\section{REFERENCES}

Carter R, Graves PM 1988. Gametocytes. In WH Wernsdorfer, I McGregor (eds.), Malaria: principles and practice of malariology, vol. I, Churchill Livingstone, Edinburgh, p. 253-303.

CDC - Centers for Disease Control and Prevention 1994. Epi Info version 6 . A word processing data base and statistics program for public health on IBM-compatible microcomputers. CDC, Atlanta, GA, USA.

Drew DR, Reece SE 2007. Development of reverse-transcription PCR techniques to analyse the density and sex ratio of gametocytes in genetically diverse Plasmodium chabaudi infections. Mol Biochem Parasitol 156: 199-209.

FMH - Federal Ministry of Health 2005. National Antimalarial Treatment Guidelines, FMH, Abuja, 33 pp.

Happi CT, Gbotosho GO, Falade CO, Akinboye DO, Gerena L, Hudson T, Sowunmi A, Kyle DE, Milhous W, Wirth DF, Oduola AMJ 2003. Point mutation in the pfcrt and pfmdr-1 genes of Plasmodium falciparum and clinical response to chloroquine among malaria patients from Nigeria. Ann Trop Med Parasitol 97: 439-451.

Happi CT, Gbotosho GO, Folarin OA, Bolaji OM, Sowunmi A, Kyle DE, Milhous W, Wirth DF, Oduola AMJ 2006. Association between mutations in pfcrt and pfmdr-1 genes and in vivo amodiaquine resistance in falciparum malaria-infected children in Nigeria. Am J Trop Med Hyg 75: 155-161.

Happi CT, Gbotosho GO, Folarin OA, Sowunmi A, Hudson T, O’Neil M, Milhous W, Wirth DF, Oduola AMJ 2009. Selection of Plas- 
modium falciparum multi-drug resistance gene 1 alleles in asexual stages and gametocytes by artemether-lumefantrine in Nigerian children with uncomplicated falciparum malaria. Antimicrob Agents Chemother 53: 888-895.

Happi CT, Gbotosho GO, Sowunmi A, Falade CO, Akinboye DO, Hudson T, Gerena L, Kyle DE, Milhous W, Wirth DF, Oduola AMJ 2004. Molecular analysis of Plasmodium falciparum recrudescent malaria infections in children treated with chloroquine in Nigeria. Am J Trop Med Hyg 70: 20-26

Mitri C, Thiery I, Bourgouin C, Paul REL 2009. Density-dependent impact of the human malaria parasite Plasmodium falciparum gametocyte sex ratio on mosquito infection rates. Proc $R$ Soc Lond Series B 276: 3721-3726.

Mokuolu OA, Okoro EO, Ayetoro SO, Adewara AA 2007. Effect of artemisinin-based treatment policy on consumption pattern of antimalarials. Am J Trop Med Hyg 76: 7-11.

Nee S, West SA, Read AF 2002. Inbreeding and parasite sex ratios. Proc R Soc Lond Series B 269: 755-760.

Okell LC, Drakeley CJ, Ghani AC, Bousema T, Sutherland CJ 2008. Reduction of transmission from malaria patients by artemisinin combination therapies: a pooled analysis of six randomized trials. Malaria J 7: 125.

Ouédraogo AL, Bousema T, Schneider P, de Vlas SJ, Iboudo-Sanogo E, Cuzin-Ouattara N, Néblé I, Roeffen W, Verhave JP, Luty AJ, Sauerwein R 2009. Substantial contribution of submicroscopical Plasmodium falciparum gametocyte carriage to the infectious reservoir in an area of seasonal transmission. PLoS ONE 4: e8140.

Pickering J, Read AF, Guerrero S, West SA 2000. Sex ratio and virulence in two species of lizard malaria parasites. Evol Ecol Res 2: $171-184$

Price RN, Nosten F, Luxemburger C, ter Kuile KO, Paiphun L, Chongsuphajasiddhi T, White NJ 1996. Effects of artemisinin derivatives on malaria transmissibility. Lancet 347: 1654-1658.

Read AF, Narara A, Nee S, Keymer AE, Day KP 1992. Gametocyte sex ratios as indirect measures of outcrossing rates in malaria. Parasitol 104: 387-395.

Reece SE, Drew DR, Gardner A 2008. Sex ratio adjustment and kin discrimination in malaria parasites. Nature 453: 609-614.

Robert V, Read AF, Essong J, Tchuinkam T, Mulder B, Verhave JP, Carnevale P 1996. Effects of gametocyte sex ratio on infectivity of Plasmodium falciparum to Anopheles gambiae. Trans $R$ Soc Trop Med Hyg 90: 621-624.

Robert V, Sokhna CS, Rogier C, Ariey F, Trape JF 2003. Sex ratio of Plasmodium falciparum gametocytes in inhabitants of Dielmo, Senegal. Parasitology 127: 1-8.
Salako LA, Ajayi FO, Sowunmi A, Walker O 1990. Malaria in Nigeria: a revisit. Ann Trop Med Parasitol 84: 435-445.

Shekalaghe SA, Bousema JT, Kunel KK, Lushino P, Masokoto A, Wolters LR, Mwakalinga S, Mosha FW, Sauerwein RW, Drakeley CJ 2007. Submicroscopic Plasmodium falciparum gametocyte carriage is common in an area of low and seasonal transmission in Tanzania. Trop Med Inter Health 12: 547-553.

Sinden RE 1983. Sexual development of malaria parasites. Adv Parasitol 22: 153-216

Sowunmi A, Balogun ST, Gbotosho GO, Happi CT 2009a. Plasmodium falciparum sex ratios in acutely symptomatic children treated with antimalarial drugs. Acta Tropica 109: 108-117.

Sowunmi A, Balogun T, Gbotosho GO, Happi CT, Adedeji AA, Fehintola FA 2007. Activities of amodiaquine, artesunate and artesunate-amodiaquine against asexual- and sexual stage parasites in falciparum malaria in children. Antimicrob Agents Chemother 51: 1694-1699.

Sowunmi A, Gbotosho GO, Happi CT, Folarin OA, Balogun ST 2009b. Population structure of Plasmodium falciparum gametocyte sex ratios in malarious children in an endemic area. Parasitol International 58: 438-443.

SPSS 1999. SPSS for Windows release 10.01 (standard version). SPSS Inc, Chicago, IL, USA.

Tchuinkam T, Mulder B, Dechering K, Stoffels H, Verhave JP, Cot M, Carnevale P, Meuwissen JEH, Robert V 1993. Experimental infections of Anopheles gambiae with Plasmodium falciparum of naturally infected gametocyte carriers in Cameroon: factors influencing the infectivity to mosquitoes. Trop Med Parasitol 44: 271-276.

West SA, Reece SE, Read AF 2001. Evolution of gametocyte sex ratios in malaria and related apicomplexan (protozoan) parasites. Trends Parasitol 17: 525-531.

West SA, Smith TG, Nee S, Read AF 2002. Fertility insurance and the sex ratios of malaria and related hemosporin blood parasites. J Parasitol 88: 258-263.

West SA, Smith TG, Read AF 2000. Sex allocation and population structure in apicomplexan (protozoa) parasites. Proc R Soc Lond Series B 267: 257-263.

WHO - World Health Organization 2000. Severe falciparum malaria. Trans R Soc Trop Med Hyg 94 (Suppl. 1): 1-90.

WHO - World Health Organization 2001. Antimalarial drug combination therapy. Report of a WHO Technical Consultation, Document WHO/CDS/RBM/2001.35, WHO, Geneva, 36 pp.

WHO - World Health Organization 2010. [acessed 7 December 2010]. Roll Back Malaria 2010. Available from: rollbackmalaria.org/ cmc-upload/0/000/015/364/RBMInfosheet-9.htm. 